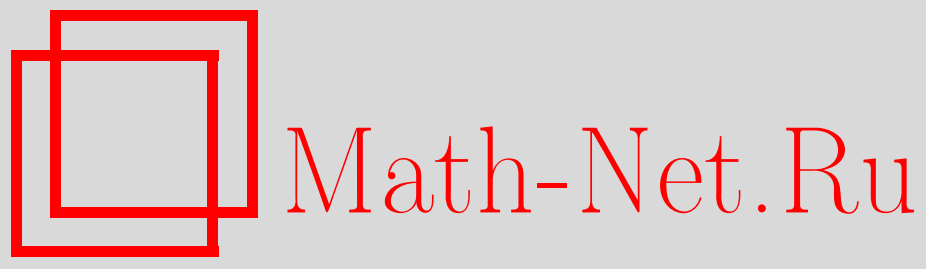

И. В. Камоцкий, М. В. Ружанский, Оценки и спектральные асимптотики для систем с кратностью, Функи. анализ и его прил., 2005, том 39, выпуск $4,78-80$

DOI: https://doi.org/10.4213/faa87

Использование Общероссийского математического портала Math-Net.Ru подразумевает, что вы прочитали и согласны с пользовательским соглашением

http://www . mathnet.ru/rus/agreement

Параметры загрузки:

IP: 54.162 .127 .20

26 апреля 2023 г., 16:17:28 


\title{
Оценки и спектральные асимптотики для систем с кратностью*
}

\author{
(c) 2005. И. В. КАмОцКИй, М. В. РУжАНСКИЙ
}

1. Введение. Пусть $X$ - гладкое многообразие без края размерности $n \geqslant 3$. Пусть $P$ - эллиптический самосопряженный псевдодифференциальный оператор первого порядка, действующий на полуплотностях на $m$-мерных сечениях векторных расслоений над $X$. Для функции $u=u(t, x)$ рассмотрим задачу Коши

$$
\left\{\begin{array}{l}
i u^{\prime}-P u=0, \quad(t, x) \in[0, T] \times X, \\
\left.u\right|_{t=0}=u_{0} .
\end{array}\right.
$$

Хорошо известно, что спектральная асимптотика оператора $P$ тесно связана с представлением пропагатора задачи (1) в виде интегрального оператора Фурье. Такое представление было получено в [4] в случае, когда задача (1) строго гиперболическая, т. е. когда характеристики (термы) оператора $P$ вещественны и попарно различны. В этом случае первый и второй члены спектральной асимптотики оператора $P$ были получены в работах [3] и [2]. В случае когда оператор $P$ имеет кратные термы $a_{j}, 1 \leqslant j \leqslant m$, и гладко диагонализуем, а также выполнено условие $\left\{a_{j}, a_{k}\right\} \neq 0$ в точках кратности $a_{j}=a_{k}$, решение задачи (1) было представлено как асимптотический ряд по гладкости в работе [7]. Члены этого ряда являются итерированными интегральными операторами Фурье, проинтегрированными по времени. Там же были исследованы спектральные асимптотики таких систем. Отметим, что некоторые результаты о втором члене спектральной асимптотики были получены также в [5] без условия гладкой диагонализуемости, но при дополнительных предположениях на распространение особенностей решений системы (1) (которых мы не налагаем).

Оценки решений в $L^{p}$ для строго гиперболических скалярных уравнений и невырожденных интегральных операторов Фурье были получены в [9]. См. также обзор [8], где оценки в $L^{p}$ были связаны с геометрическими свойствами и особенностями волновых фронтов соответствующих интегральных операторов Фурье.

Сделаем следующие предположения. Предположим, что главный символ оператора $P$ гладко микролокально диагонализуем, т. е. микролокально имеет гладкие собственные пространства и гладкие собственные значения $a_{j}$. Предположим также, что существует число $M$, такое, что для всех $(x, \xi) \in T^{*} X$ и всех $1 \leqslant j, k \leqslant m, j \neq k$, таких, что $a_{j}$ и $a_{k}$ не совпадают тождественно, в точках $a_{j}(x, \xi)=a_{k}(x, \xi)$ для итерированных скобок Пуассона выполнено условие

$$
\left\{a_{j},\left\{a_{j}, \cdots\{a_{j}, a_{k} \overbrace{\}\} \cdots\}}^{\lambda}(x, \xi) \neq 0,\right.\right.
$$

*Работа поддержана EPSRC, грант GR/R67583/01. 
для некоторого $\lambda(x, \xi) \leqslant M$. Строго гиперболический случай соответствует $M=0$. Трансверсальный случай Розенблюма в [7] соответствует $M=1$. Случай скользящих поверхностей Мелроуза [6] соответствует $M=2$. Отметим, что условие (2) описывает указанные системы в общем положении.

2. Формулировка результатов. Оказывается возможным установить (в целом точные) оценки в $L^{p}$ для решений задачи (1). Через $L_{\alpha}^{p}=W^{p, \alpha}$ обозначим обычное пространство Соболева над $L^{p}$ с $\alpha \in \mathbb{R}$ производными.

Teорема 1. Пусть $1<p<\infty u \alpha=(n-1)|1 / p-1 / 2|$. Пусть $P=P\left(t, x, D_{x}\right)$ есть $m \times m$-матрища, составленная из классических эллиптических псевдодифберенциальных операторов первого порядка. Пусть

$$
P\left(t, x, D_{x}\right)=A\left(x, D_{x}\right)+B\left(t, x, D_{x}\right),
$$

где $A$ - симметричная матрица из псевдодифберенциальных операторов первого порядка, а $B$ - матрица из операторов нулевого порядка, гладко зависящux от $t$. Предположим, что матрииа $A$ удовлетворяет условию (2). Тогда для всех функиий $f \in L_{\alpha}^{p} \cap L^{2}$ с компактным носителем решение $u=u(t, x)$ задачи Коши

$$
i \frac{\partial u}{\partial t}-P\left(t, x, D_{x}\right) u=0, \quad u(0)=f,
$$

принадлежнт $L_{\mathrm{loc}}^{p}$ для всех $0<t \leqslant T$ при некотором $T>0$. Более того, найдется такое $C>0$, что выполнено неравенство $\sup _{0 \leqslant t \leqslant T}\|u(t, \cdot)\|_{L^{p}} \leqslant$ $C_{T}\|f\|_{L_{\alpha}^{p}}$.

Условие гладкой микролокальной диагонализуемости матрицы $A$ выполнено в ряде важных случаев. Так, некоторые системы Максвелла гладко диагонализуемы (см. [1]). Более того, слабо гиперболические скалярные операторы с инволютивными характеристиками могут быть приведены к системе, удовлетворяющей условиям теоремы 1.

Обозначим через $\Phi_{J}(T, x, \xi)$ гамильтонов поток по ломаным траекториям системы. При $J=\left(j_{1}, \ldots, j_{k}\right)$ в момент $T$ это поток из $(x, \xi)$ по бихарактеристике $a_{j_{1}}$ до точки пересечения $a_{j_{1}}=a_{j_{2}}$, затем поток по бихарактеристике $a_{j_{2}}$ и т. д. Для упрощения формулировки предположим сейчас, что термы $a_{k}$ положительны. Если имеются также и отрицательные термы, то с помощью псевдодифференциальных проекторов можно исследовать каждую из задач в отдельности.

Теорема 2. Предположим, что многообразие $X$ компактно и что выполнено условие (2). Обозначим через $D$ множество тех $(x, \xi) \in T^{*} X$, для котоpых существуют $T$ u J, такие, что $\Phi_{J}(T, x, \xi)=(x, \xi)$. Предположсим, что $D$ имеет нулевую меру. Тогда спектр оператора $P$ имеет вейлевскую асимпmomuкy

$$
N(\lambda)=\#\left\{j: \lambda_{j}<\lambda\right\}=c_{n} \lambda^{n}+c_{n}^{\prime} \lambda^{n-1}+o\left(\lambda^{n-1}\right),
$$

где $\lambda_{j}$ - собственные значения оператора $P$.

Основными составляющими доказательства являются представление решения задачи (1) в виде асимптотического ряда, исследование свойств членов этого ряда и доказательство их сглаживающих свойств. Основная техническая сложность по сравнению с [7] - это доказательство сглаживания при $M>1$. 
Теорема 2 выводится из полученного представления. Теорема 1 требует дополнительного анализа остатка ряда, а также использования некоторых фактов из функционального анализа и теории функций.

3. Обсуждение. 1. Условие (2) означает, что бихарактеристики, соответствующие терму $a_{j}$, пересекают поверхность уровня $a_{k}=1$ с порядком, не превышающим $M$.

2. Оценки решений теоремы 1 совпадают с оценками решений строго гиперболических уравнений. В общем случае потеря $(n-1)|1 / p-1 / 2|$ производных в $L^{p}$ является точной, например, в случае, когда размерность сингулярного носителя интегрального ядра разрешающего оператора равна $2 n-1$. В работе [8] эти оценки улучшены в ряде случаев с учетом геометрии волновых фронтов пропагаторов. Подобные оценки важны в исследовании нелинейных уравнений и других задачах гармонического анализа.

3. С помощью асимптотического представления решения задачи (1) и методов гармонического анализа, из $L^{p}$-оценок, полученных в теореме 1 , выводятся $L^{p}$ - $L^{q}$-оценки, а также оценки в пространствах Липшица, Харди и т. д. (см. [8]).

4. Отметим, что оценки теоремы 1 можно получить также для оператора $A$, зависящего от времени. Однако условие (2) меняется и не имеет очевидной геометрической интерпретации.

\title{
ЛитеРАТУРА
}

1. Бирман М. Ш., Соломяк М. З. УМН, 42, вып. 6 (258), 61-76 (1987). 2. Duistermaat J. J., Guillemin V. Invent. Math., 29, 39-79 (1975). 3. Hörmander L. Acta Math., 121, 193-218 (1968). 4. Hörmander L. Acta Math., 127, 79-183 (1971). 5. Иврий В. Я. Функц. анализ и его прил., 16, 101-108 (1982). 6. Melrose R. Invent. Math., 37, 165191 (1976). 7. Розенблюм Г. Зап. научн. семин. ЛОМИ, 96, 255-271, 311-312 (1980). 8. Ружанский M. B. УМH, 55, 99-170 (2000). 9. Seeger A., Sogger C. D., Stein E. M. Ann. of Math., 134, 231-251 (1991).

C.-Петербургское отделение Математического

института им. В. А. Стеклова,

Imperial College, London

e-mail: i.kamotski@imperial.ac.uk

Imperial College, London

e-mail: ruzh@ic.ac.uk

УДК 517.9

\section{Асимптотический вариант общих теорем о коммутаторах}

\author{
(C) 2005. М. И. КАРАХАНЯН
}

1. Известная теорема Фугледе устанавливает, что если нормальный ограниченный оператор $c$ гильбертова пространства коммутирует с некоторым ограниченным оператором $x$, то он коммутирует и с сопряженным с ним оператором. В таком виде эта теорема давно входит в учебники по функциональному анализу (см., например, [1]). У этой теоремы, имеющей давнюю историю, имеются, в

*Работа поддержана EPSRC, грант GR/R67583/01. 